\title{
First nitrogen-seeding experiments in JET with the ITER-like Wall
}

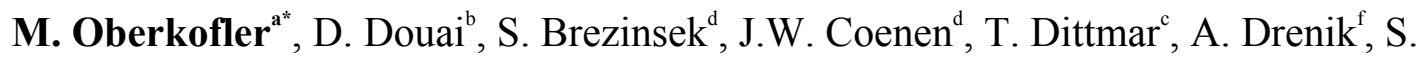

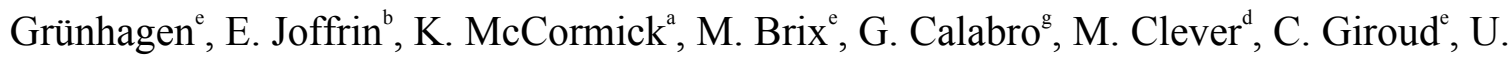

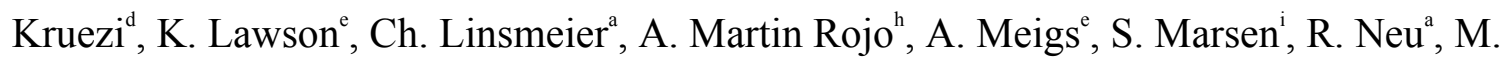

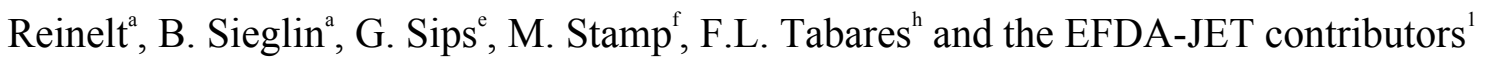
JET-EFDA, Culham Science Centre, Abingdon, OX14 3DB, UK

${ }^{a}$ Max Planck Institute for Plasma Physics, EURATOM Association, Boltzmannstr. 2, 85748 Garching, Germany

${ }^{b}$ CEA Centre de Cadarache, 13108 Saint Paul lez Durance Cedex, France

${ }^{c}$ Center for Energy Research, University of California-San Diego, 9500 Gilman Dr., San Diego, CA 92093-0417, USA

${ }^{d}$ Institut für Energie- und Klimaforschung, IEK-4, TEC, Association EURATOM-FZJ, 52425 Jülich,

Germany

${ }^{e}$ EURATOM/CCFE Fusion Association, Culham Science Centre, Abingdon, Oxon, OX14 3DB, UK

${ }^{f}$ Jožef Stefan Institute, Jamova 39, 1000 Ljubljana, Slovenia

${ }^{g}$ Associazione EURATOM-ENEA sulla Fusione, Via E. Fermi 45 FRASCATI-Roma, Italy

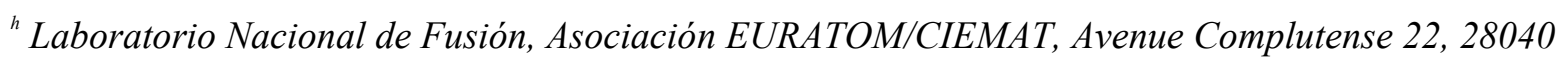
Madrid, Spain

${ }^{i}$ Max Planck Institute for Plasma Physics, EURATOM Association, Wendelsteinstr. 1, 17491

Greifswald, Germany

\begin{abstract}
In this contribution we present results from the first $\mathrm{N}_{2}$ seeding experiments in JET performed after installation of the ITER-like Wall. Gas balance measurements for seeded L-mode discharges indicate very strong $\mathrm{N}_{2}$ retention as well as a potential increase in $\mathrm{D}_{2}$ retention. The

\footnotetext{
${ }^{1}$ See App. of F. Romanelli et al., Proc. of the $23^{\text {rd }}$ IAEA Fusion Energy Conf. 2010, Daejon, Korea.
} 
possible influence of ammonia production on this apparent retention is discussed. Plasma parameters and impurity content were monitored throughout the seeded discharges as well as during subsequent clean-up discharges. These experiments give first insight into phenomena related to the use of nitrogen as seeding gas in JET with the ITER-like Wall, such as ammonia production and nitrogen legacy.

PACS: 52.40.Hf, 52.55.Fa, 52.55.Rk

PSI-20 keywords: Retention mechanisms, Impurity seeding, Ion-surface interactions, JET-ILW "Corresponding author address: MPI für Plasmaphysik, Boltzmannstr. 2, 85748 Garching b. München, Germany

"Corresponding author e-mail: martin.oberkofler@ipp.mpg.de

Presenting author: Martin Oberkofler

Presenting author e-mail: martin.oberkofler@ipp.mpg.de 


\section{Introduction}

The reduction of power loads on the tungsten divertor target of ITER is mandatory in relevant heated scenarios. To this aim it is foreseen to seed noble gases or nitrogen into the divertor plasma. The seeding species will interact with the plasma-facing materials beryllium and tungsten. In the case of nitrogen also chemical reactions with the wall surfaces as well as with hydrogen have to be considered. The efficiency and the side effects of $\mathrm{N}_{2}$ seeding in a tokamak with a first wall constituted of Be and $\mathrm{W}$ are currently being studied at JET with its ITER-like Wall (ILW) $(1 ; 2)$. In this paper we present results from the first experiment with $\mathrm{N}_{2}$ injection in JET-ILW. The main aim of this experiment was to assess the nitrogen retention and the influence of $\mathrm{N}_{2}$ injection on the retention of hydrogen isotopes. Furthermore, the impurity content in the vessel was monitored at various $\mathrm{N}_{2}$ injection levels as well as during subsequent clean-up discharges.

\section{Gas balance}

The chosen scenario for the gas balance with $\mathrm{N}_{2}$ seeding was a standard L-mode with low triangularity, ohmic heating, 2.0 MA plasma current and 2.2 T toroidal field. $\mathrm{N}_{2}$ was injected into the divertor scrape-off layer through the toroidally symmetric gas injection ring (GIM9) close to the horizontal $\mathrm{W}$ target plate on which the outer strike-point was positioned. The core density in the flat top phase was $2.5 \cdot 10^{19} \mathrm{~m}^{-3}$ in the first discharges. It was lowered in later discharges to $1.8 \cdot 10^{19} \mathrm{~m}^{-3}$ to be able to inject more $\mathrm{N}_{2}$ without running into a radiation limit. The plasma-facing surfaces were loaded with nitrogen by steadily increasing the $\mathrm{N}_{2}$ injection with each discharge. Injection rates between 1.4 and $5.4 \cdot 10^{21}$ electrons per second and ratios of $\mathrm{N}_{2}$ to $\mathrm{D}_{2}$ (in molecules per second) from 0.04 to 1.3 were covered. Before the experiment, a liquid helium (LHe) cryo regeneration was performed in order to collect only the gas consumed in this experiment. After 13 successful discharges the LHe cryo panel of the pumped divertor was regenerated again. The released gas was transferred to the active gas handling system (AGHS) where its total volume and composition was measured. Subsequent to this 
loading session, a series of clean-up discharges without $\mathrm{N}_{2}$ injection was performed with the aim to remove nitrogen stored in the plasma-facing surfaces. These included limiter discharges with scans of the radial gaps, divertor discharges with large strike-point sweeps as well as divertor discharges with additional $2 \mathrm{MW}$ of ion cyclotron heating. After 18 clean-up discharges the LHe cryo panel was regenerated once more and the gas analyzed in the AGHS.

The results of the two gas balances are shown in Table 1. The most striking evidence is that about half of the injected $\mathrm{N}_{2}$ was not recovered in the AGHS upon the first regeneration of the LHe cryo panel. This is in accordance with the strong gas consumption observed with the CFC-wall after heavy Be evaporation (3). The percentage numbers for the apparent retention of $\mathrm{D}$ and total amount of gas have to be compared to non-seeded L-mode discharges at a similar density. In the ILW-configuration the total retention (corresponding roughly to the $\mathrm{D}_{2}$ retention) is typically around $1-2 \%$ in similar discharges, corresponding to retention rates of about $5 \cdot 10^{19} \mathrm{D} / \mathrm{s}$ normalised to the divertor phase $(4 ; 5)$. Thus, the apparent overall retention is considerably increased by the $\mathrm{N}_{2}$ injection. In the extensive series of non-seeded cleaning discharges only $3 \cdot 10^{20} \mathrm{~N}$ atoms were injected in a short blib (for determination of the pumping speed), while 11 times more was recovered upon regeneration of the LHe cryo panel. (Mathematically this yields the high negative percentage of missing $\mathrm{N}_{2}$ listed in the table.) However, this amounts to merely $16 \%$ of the $\mathrm{N}_{2}$ that was missing after the first regeneration.

Laboratory experiments have shown that the amount of nitrogen retained upon implantation into Be or W saturates very quickly $(5 ; 6)$. The surface layer thickness within which nitrogen is retained corresponds to the range of the energetic $\mathrm{N}$ particles in the solid and is therefore limited to a few $\mathrm{nm}$. In this thin layer nitrogen atomic fractions up to 0.5 are reached, consistent with the formation of a surface layer of nitride $\left(\mathrm{Be}_{3} \mathrm{~N}_{2}\right.$ and $\left.\mathrm{WN}\right)$. No diffusion of $\mathrm{N}$ into the bulk of the metals was observed. This allows a simple estimation of the amount of $\mathrm{N}$ that can be stored as nitride after implantation into plasma-facing materials. To first order, the 
contribution of the $\mathrm{W}$ surfaces in the divertor is neglected due to their smaller area and the shallower penetration depth of energetic $\mathrm{N}$ atoms in the high-Z material. Assuming a $\mathrm{Be}$ surface area of $200 \mathrm{~m}^{2}$ and a penetration depth of $10 \mathrm{~nm}$ (corresponding to an implantation energy of a few $\mathrm{keV}$ ) and a density of $\mathrm{Be}_{3} \mathrm{~N}_{2}$ of $2.7 \mathrm{~g} / \mathrm{cm}^{3}$ (7) leads to $6 \cdot 10^{22} \mathrm{~N}$ atoms that can be stored as beryllium nitride on plasma-facing surfaces. This compound is very stable (with a higher melting temperature than metallic $\mathrm{Be}(8)$ ) and can efficiently store the implanted nitrogen.

While this estimation indicates the order of magnitude, it is a strong simplification. Saturation of a surface layer with nitrogen might occur only on the surfaces exposed to the highest ion fluxes, i.e. the limiters. This leads to a much reduced relevant Be area. Furthermore, the simultaneous bombardment of these surfaces with energetic D leads to sputter-erosion of the nitride layer, thereby further reducing the amount of retained nitrogen. Therefore, $6 \cdot 10^{22} \mathrm{~N}$ atoms is rather an upper estimate of the net amount of nitrogen that can be stored in plasmafacing surfaces in JET. On the other hand, significant amounts of nitrogen can also be stored in co-deposits. Typical N/Be ratios in the range of 0.1-0.2 were observed in co-deposits produced in the PISCES B laboratory (9). Co-deposition with carbon can be neglected: The amount of C in the ILW-vessel is reduced by an order of magnitude with respect to JET-CFC (10). In accordance with this finding, no $\mathrm{CN}$ molecules were observed by spectroscopy.

A similar estimation for experiments performed at ASDEX Upgrade has lead to the conclusion that the assumed area of its $\mathrm{W}$ surfaces is not large enough to explain the observed $\mathrm{N}$ retention (11). An increased effective surface area due to rough surfaces was proposed as a possible reason for the observed discrepancy. However, more recent investigations on the composition of the pumped gas in $\mathrm{N}_{2}$-seeded and subsequent discharges have revealed a strong influence of ammonia formation on the gas balance (12). It is known that metal surfaces can act as efficient catalysts for the formation of ammonia from hydrogen and nitrogen $(13 ; 14)$. In the here 
presented experiments any ammonia produced will not appear in the gas balance since at liquid nitrogen temperatures it has a vapor pressure of $10^{-11}$ mbar. It is, therefore, pumped by the liquid nitrogen cryo panel and cannot be recovered upon regeneration of only the LHe panel. Ammonia production, therefore, needs to be considered a possibly important contribution to the observed $\mathrm{N}$ retention also in JET.

Form the gas balance results in table 1 the maximum amount of $\mathrm{ND}_{3}$ produced in the $\mathrm{N}_{2}$-seeded discharges can be estimated. This amount is limited by the missing deuterium $\left(3.5 \cdot 10^{22} \mathrm{D}\right.$ atoms) to $1.2 \cdot 10^{22}$ ammonia molecules. This means that a maximum of roughly $30 \%$ of the injected $\mathrm{N}$ atoms can have been converted to ammonia. For a more conservative estimate it can be assumed that the 'normal' $\mathrm{D}_{2}$ retention mechanisms, that in similar non-seeded discharges lead to a $\mathrm{D}_{2}$ retention of up to $2 \%$, are not affected by the $\mathrm{N}_{2}$ injection. The observed increase in $\mathrm{D}_{2}$ retention by $1.7 \%\left(1.6 \cdot 10^{22} \mathrm{D}\right.$ atoms $)$ would still correspond to the formation of $0.5 \cdot 10^{22}$ ammonia molecules (roughly $15 \%$ of the injected $\mathrm{N}$ atoms converted to $\mathrm{ND}_{3}$ ).

\section{Indications of ammonia production}

\section{$\underline{\text { Visible spectroscopy }}$}

The mirror-link spectroscopy system was used to record line radiation emitted by the plasma in the visible wavelength range during $\mathrm{N}_{2}$-seeded discharges. It can detect emissions from ND radicals, which feature a distinct band emission at $336.0 \mathrm{~nm}$. The formation of these molecules is a sign of ongoing plasma and/or surface chemical reactions and would be an indication also for $\mathrm{ND}_{3}$ production (14). The lines-of-sight of the mirror-link spectrometer cover the whole horizontal $\mathrm{W}$ target. Figure 1 shows a clear signal peak at $336.0 \mathrm{~nm}$ during $\mathrm{N}_{2}$ seeding. In

Figure 2 the integrated intensity of this signal is color-coded and plotted as a function of time and position along the horizontal target. The position of the outer strike-point is indicated. The 
signal from ND molecules is localized around the projection of the strike-point. Its temporal evolution is strongly correlated to that of the NII intensity at $500.5 \mathrm{~nm}$.

\section{$\underline{\text { Residual gas analysis }}$}

While visual spectroscopy indicates the formation of ND molecules, which in turn suggests the production also of $\mathrm{ND}_{3}$, it has proven challenging to confirm this with in-situ mass spectrometry. Time resolved signals at several mass-to-charge ratios including $28\left(\mathrm{~N}_{2}\right)$ as well as $18\left(\mathrm{ND}_{2}\right), 19\left(\mathrm{ND}_{2} \mathrm{H}\right)$, and $20\left(\mathrm{ND}_{3}\right)$ were recorded in the pump duct below the outer divertor. Shot-integrated partial pressures were derived by integrating the backgroundsubtracted signals over the duration of each discharge. There is a clear correlation between the partial pressure at the $\mathrm{m} / \mathrm{e}$ ratio of 28 and the injected amounts of $\mathrm{N}_{2}$. With a known pumping speed (measured for $\mathrm{N}_{2}$ without plasma) this can be converted into gas consumption, which is plotted in the top graph of Figure 3 as a function of the integral amount of $\mathrm{N}_{2}$ injected in the discharge. A linear fit to the calculated $\mathrm{N}_{2}$ consumption yields a value of 0.46 in reasonable agreement with the overall amount of $\mathrm{N}_{2}$ missing in the loading session (see Table 1). The bottom graph shows the shot-integrated partial pressures for mass to charge ratios indicative of (deuterated) ammonia. Here no correlation is observed. This seems to contradict the strong ammonia production that is indicated by the global gas balance and the spectroscopy measurements. This discrepancy might be due to contributions to the mass spectrometer signals from (deuterated species of) water and/or methane (12).

\section{Nitrogen legacy}

The shot-to-shot legacy of nitrogen in the vacuum vessel after an $\mathrm{N}_{2}$-seeded discharge used to pose an operational difficulty in JET with the carbon wall (3): The nitrogen content in the edge plasma gradually increased from shot to shot, making it rather challenging to execute two equivalent $\mathrm{N}_{2}$-seeded discharges in a row. With the new ITER-like Wall this issue is less compromising: In the seeded phase of each discharge the $\mathrm{N}$ impurity levels and plasma 
parameters are not affected by the amount of $\mathrm{N}_{2}$ injected in previous discharges. This is exemplified in Figure 4 for the case of core radiation. This graph shows that the bolometric signal increases during the $\mathrm{N}_{2}$-seeded phase of each discharge. This correlation with the injected amount up to the measurement point indicates a buildup of the inventory of the radiating species. However, the data points for various discharges fall on top of each other. This means that the observed radiation is independent of the injection in the previous discharge.

In conclusion, Figure 4 shows that no nitrogen legacy is observed when comparing seeded phases of various discharges. However, clear signs of a shot-to-shot nitrogen legacy were monitored in a non-seeded reference phase at the beginning of each discharge. Figure 5 shows the shot-to-shot evolution of the NII line intensity at $500.5 \mathrm{~nm}$ at the position of the outer strike-point in this non-seeded reference phase. The nitrogen recycling flux around the outer strike-point increases with increasing amount of $\mathrm{N}_{2}$ injected in the foregoing discharge and drops again steadily in the non-seeded discharges.

\section{Conclusions}

Nitrogen-seeded L-mode discharges were successfully run at JET with the ITER-like Wall. A gas balance resulted in very strong apparent $\mathrm{N}_{2}$ retention and increased $\mathrm{D}_{2}$ retention. The influence of the formation of deuterated ammonia molecules on this gas balance could not be quantified. However, we conclude that considerable amounts of ammonia can be produced. This must be taken into account in the discussion to use $\mathrm{N}_{2}$ as an extrinsic radiator in ITER and potentially in the design of the ITER tritium plant. The decomposition of mass spectra into contributions from various molecules has enabled a quantification of ammonia production at AUG (12). A similar analysis should be attempted at JET in the future.

A considerable contribution to the observed nitrogen consumption from implantation of $\mathrm{N}$ into plasma-exposed surfaces could not be excluded. Further gas balance experiments are therefore 
desirable on more strongly seeded discharges, where this retention mechanism is expected to saturate.

A clear shot-to-shot nitrogen legacy is observed spectroscopically in the non-seeded reference phase of the discharges. The legacy strongly decays from shot to shot in non-seeded discharges. However, the evolution of impurity content and plasma parameters in the seeded phase of each discharge is not influenced by the amount of $\mathrm{N}_{2}$ injected in preceding discharges. This relaxes an operational difficulty previously encountered with the carbon wall. A plausible reason for this reduced legacy is a reduced adsorption of gaseous impurities: The effective surface area available for adsorption is much smaller for metallic plasma facing materials compared to porous CFC components.

\section{Acknowledgements}

This work was supported by EURATOM and carried out within the framework of the European Fusion Development Agreement. The views and opinions expressed herein do not necessarily reflect those of the European Commission.

\section{References}

1. Philipps, V. et al., Fus. Eng. Des. 85 (2010) 1581

2. Matthews, G.F. et al., Phys. Scr. T138 (2009) 014030

3. Brezinsek, S. et al., Nucl. Fusion 51 (2011) 073007

4. Loarer, T. et al., submitted to PSI-20 (2012)

5. Brezinsek, S. et al., submitted to IAEA (2012)

6. Oberkofler, M. and Linsmeier, Ch., Nucl. Fusion 50 (2010) 125001

7. Schmid, K. et al. Nucl. Fusion 50 (2010) 025006

8. Rabenau, P. and Eckerlin A., 1960, Zeitschrift für anorganische und allgemeine Chemie 304 (1960) p. 220 
9. Okamoto, H. and Tanner, L., Phase Diagrams of Binary Beryllium Alloys; Monograph

Series on Alloy Phase Diagrams. s.l.: Materials Park, OH: ASM International (1987) p. 123

10. Dittmar, T. et al., submitted to PSI-20 (2012)

11. Brezinsek, submitted to PSI-20 (2012)

12. Kallenbach, A. et al., Plasma Phys. Control. Fus. 52 (2010) 055002

13. Neuwirth, D. et al., accepted by Plasma Phys. Control. Fus. (2012)

14. van Helden, J.H. et al., J. Appl. Phys. 101 (2007) 043305

15. O. Kiyooka and H. Matsumoto, Plasma Chem. Plasma P. 16,4 (1996) 547 


\section{Table captions}

Table 1: Results of the two gas balances performed after the series of $\mathrm{N}_{2}$-seeded discharges and after the subsequent cleaning discharges. Injected amounts are given in terms of numbers of atoms.

\section{Figure captions}

Figure 1: Line radiation from ND molecules observed along a line of sight looking at the outer strike point.

Figure 2: Contour plots of ND and NII signals vs time and position along the horizontal target.

Figure 3: Top: Shot-integrated $\mathrm{N}_{2}$ consumption (injected minus pumped) for all $\mathrm{N}_{2}$ seeded discharges as a function of total amount of $\mathrm{N}_{2}$ injected within the discharge. Bottom: Shotintegrated partial pressures for the mass-to-charge ratios that could be a signature for (deuterated) ammonia.

Figure 4: Core radiation from bolometric measurements during the $\mathrm{N}_{2}$-seeding phase of various discharges as a function $\mathrm{N}_{2}$ injected up to the point of measurement.

Figure 5: Evolution of the NII line radiation at $500.5 \mathrm{~nm}$ and of the effective charge $\mathrm{Z}_{\text {eff }}$ from shot to shot. The logarithm of the line intensity along a line of sight looking onto the outer divertor target close to the outer strike-point was averaged over a time window with no $\mathrm{N}_{2}$ injection at the beginning of each discharge. 


\section{Tables}

Table 1

\begin{tabular}{|l|r|r|r|r|}
\hline \multirow{2}{*}{} & \multicolumn{2}{|l|}{ Loading } & \multicolumn{2}{l|}{ Removal } \\
\cline { 2 - 5 } & injected & missing & injected & \multicolumn{1}{l|}{ missing } \\
\hline $\mathrm{N}$ & $3.8 \cdot 10^{22}$ & $52.5 \%$ & $3 \cdot 10^{20}$ & $-1100.0 \%$ \\
\hline $\mathrm{D}+\mathrm{H}$ & $0.97 \cdot 10^{24}$ & $3.7 \%$ & $1.07 \cdot 10^{24}$ & $1.4 \%$ \\
\hline total & $1.01 \cdot 10^{24}$ & $5.5 \%$ & $1.07 \cdot 10^{24}$ & $1.1 \%$ \\
\hline
\end{tabular}


Figures

Figure 1

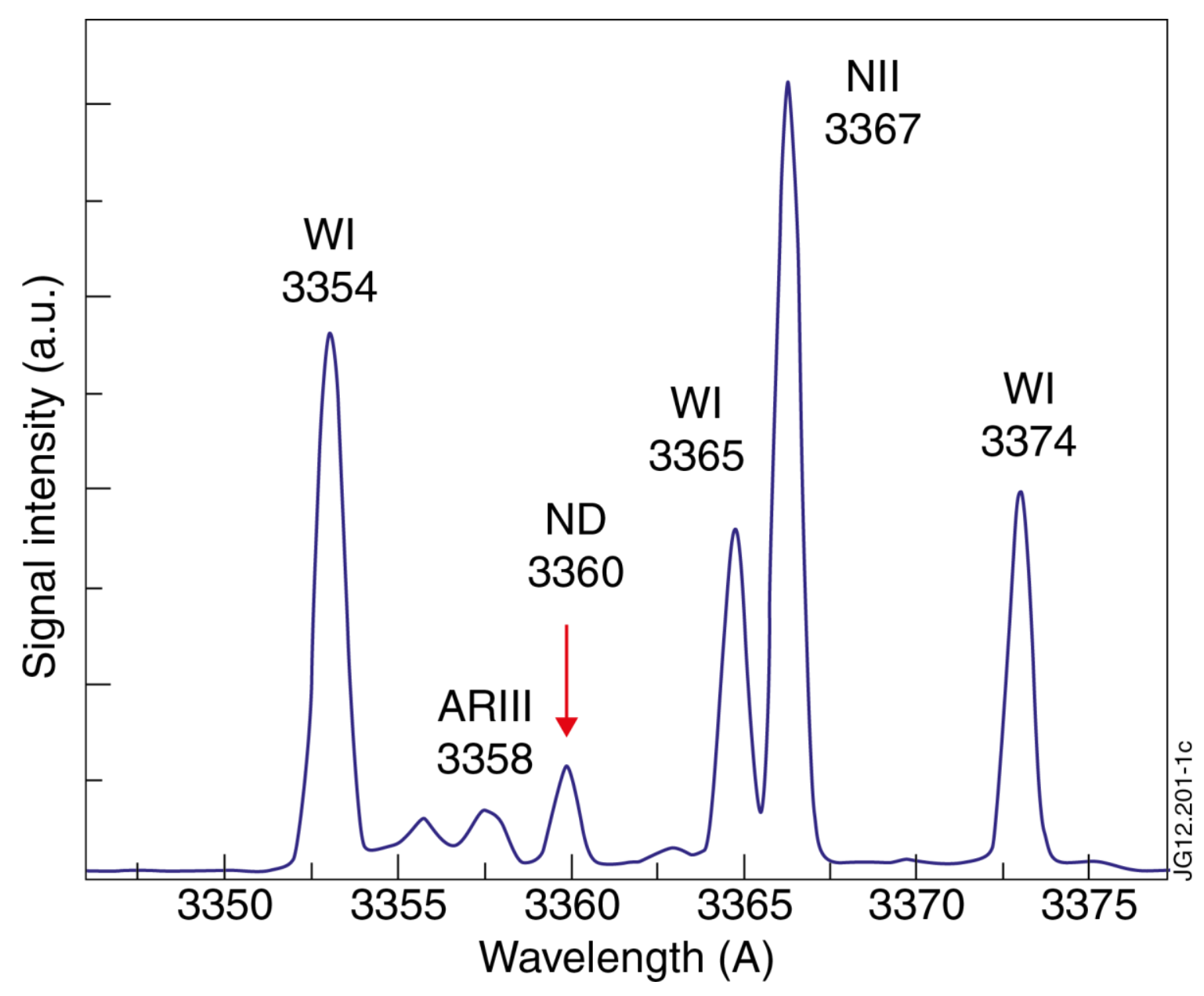


Figure 2

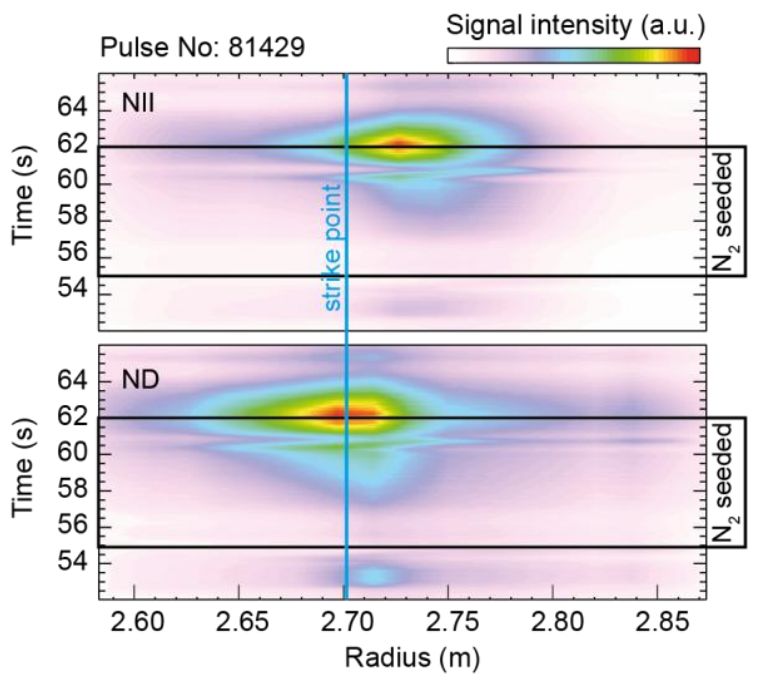


Figure 3

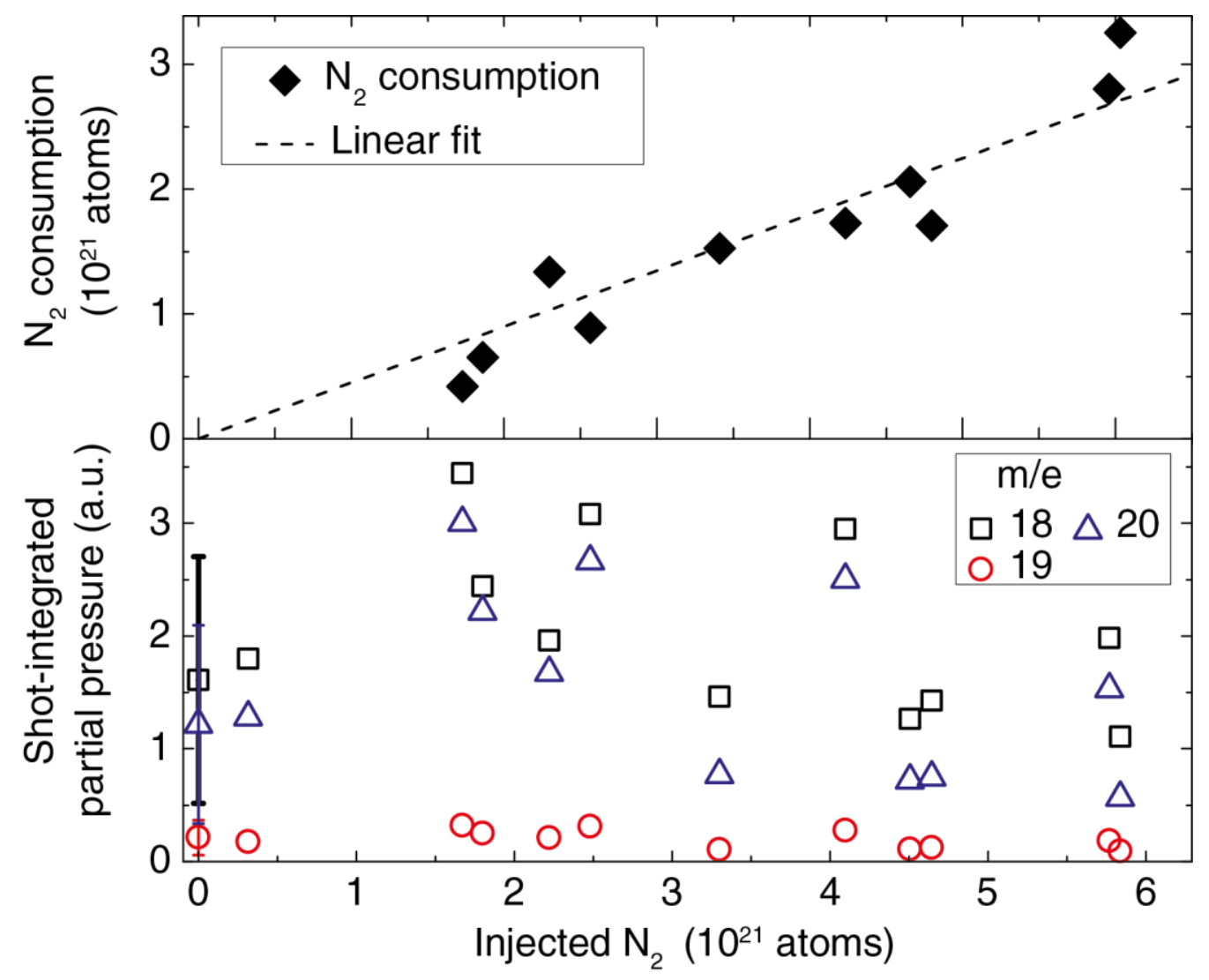


Figure 4

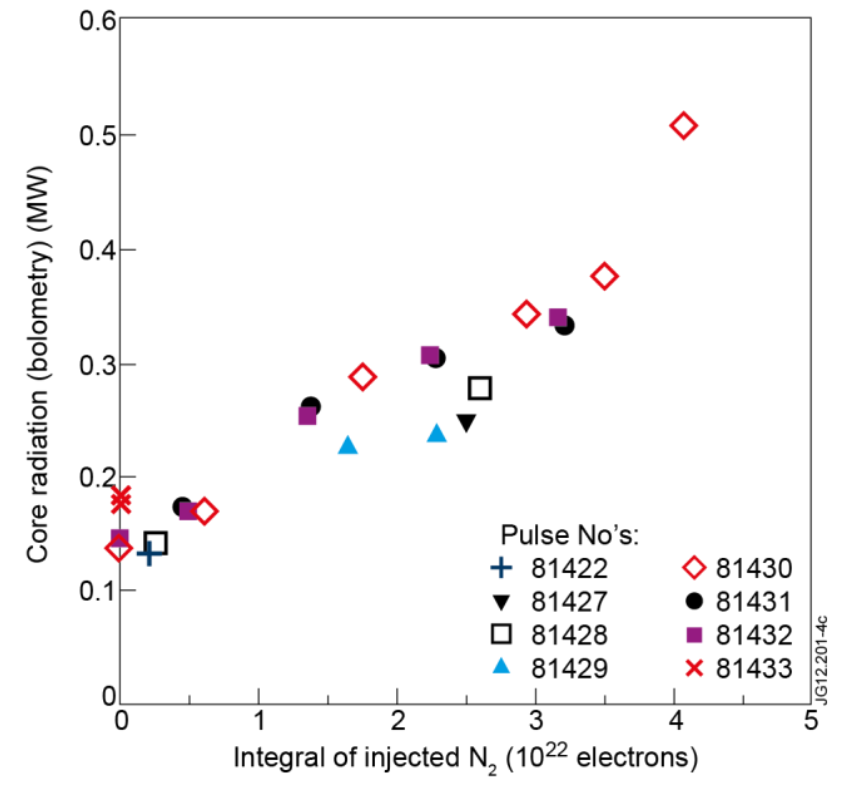


Figure 5

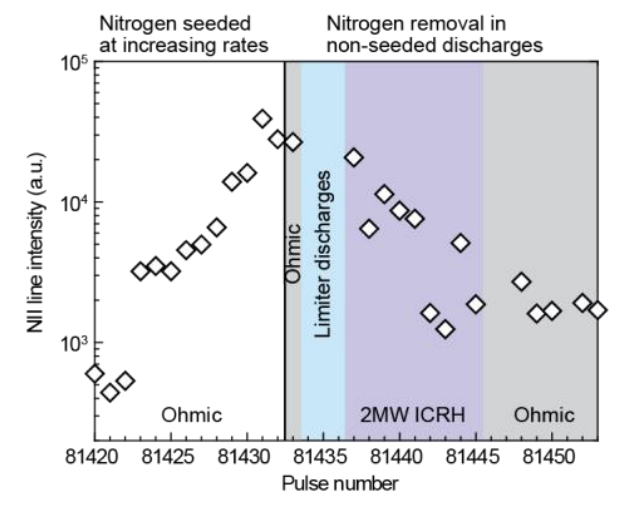

